\title{
SUPLEMENTAÇÃO COM VITAMINA $C$ E $E$ SOBRE O DESENVOLVIMENTO E SOBREVIVÊNCIA DE GIRINOS DE RÃ-TOURO (Rana catesbeiana Shaw, 1802)
}

\author{
VITAMIN C AND E SUPPLEMENTATION ON THE DEVELOPMENT AND SURVIVAL OF \\ BULLFROG TADPOLES (Rana catesbeiana Shaw, 1802)
}

\author{
Marta Verardino De Stéfani ${ }^{1}$ Adriana Sacioto Marcantonio ${ }^{2}$ \\ Maurício Laterça Martins ${ }^{3}$
}

RESUMO

$O$ presente trabalho teve como objetivo avaliar os efeitos da suplementação de vitamina C e E sobre o desenvolvimento e sobrevivência de girinos de rã-touro. Os animais receberam 50, 250 e 500mg de vitamina $\mathrm{C}$ ou $\mathrm{E} / \mathrm{kg}$ de ração, distribuídos segundo delineamento inteiramente casualizado com 3 repetições cada. $\mathrm{Na}$ dieta controle, não foram adicionadas vitaminas. Os girinos suplementados com vitamina $C$ ou $E$ não apresentaram diferença significativa no crescimento, comparados ao controle. Não obstante, os suplementados com 500mg de vitamina C apresentaram melhor sobrevivência $(62,83 \%)$ do que os animais controle $(40,48 \%$; $p<0,05)$, nos demais níveis desta vitamina e a adição de vitamina $E$ não alteraram a sobrevivência. $O$ presente trabalho sugere que $500 \mathrm{mg}$ de vitamina $\mathrm{C} / \mathrm{kg}$ de ração constitui uma opção para melhorar a sobrevivência de girinos de rã-touro.

Palavras-chave: vitamina $C$, vitamina $E$, rã-touro, Rana catesbeiana, sobrevivência.

\section{SUMMARY}

The effects of vitamin $C$ and $E$ supplementation on the development and survival of bullfrog tadpoles were evaluated. The animals received 50, 250 and 500mg vitamin Cor E/kg dry ration. In the control diet, vitamins were not added. A fully randomized experimental design was utilized, with 3 replicates of each treatment. Vitamin supplemented tadpoles did not show significant difference on growth compared to the control. Furthermore, tadpoles with supplementation of 500mg vitamin $C$ showed better survival (62.83\%) than control (40.48\%; $p<0.05)$. The other levels of vitamin $C$ and $E$ did not affect the survival of tadpoles. The present study suggests that $500 \mathrm{mg}$ vitamin C/kg dry ration is a tool to improve the survival of bullfrog tadpoles.

Key words: vitamin $C$, vitamin $E$, tadpoles, Rana catesbeiana, survival.

\section{INTRODUÇÃO}

A vitamina $\mathrm{C}$ contribui para a formação do tecido ósseo e cartilaginoso, sendo responsável por melhoras significativas no crescimento de peixes, e sua falta ou deficiência na dieta pode provocar deformações ósseas, branquiais, hemorragias, falta de apetite e aumento do estresse (HALVER, 1995). Em peixes, os sinais típicos de deficiência de vitamina $\mathrm{C}$ podem ser lordose, escoliose, hemorragias, baixo crescimento e perda de apetite (HALVER et al., 1975; AGRAWAL \& MAHAJAN, 1980). No único trabalho encontrado na literatura com girinos, LEIBOVITZ et al. (1982) observaram que a vitamina $\mathrm{C}$ é necessária em dietas de girinos, pois sua deficiência causa deformações na cauda dos mesmos. Em uma espécies de peixe brasileiro (Piaractus mesopotamicus), MARTINS (1995) observou que a falta de vitamina C provoca alterações estruturais como hiperplasia, espessamento das lamelas primárias e secundárias e infiltrado inflamatório mononuclear na extremidade dos filamentos branquiais. Mais tarde, MARTINS (1998) observou significativa diminuição no número de Anacanthorus penilabiatus (Monogenea) nas brânquias de $\boldsymbol{P}$. mesopotamicus alimentados com 200mg de ácido ascórbico/kg de ração durante 24 semanas.

Para demonstrar a eficácia da combinação de vitamina $\mathrm{C}$ e vitamina $\mathrm{E}$ na dieta de Oncorhynchus. mykiss, WAHLI et al. (1998)

\footnotetext{
${ }^{1}$ Docente do Centro de Aquicultura da Universidade Estadual Paulista (UNESP) - CAUNESP, doutorado em Aquicultura, Via de Acesso Prof. Paulo D. Castellane, Zona Rural, s/n, 14884-900, Jaboticabal, SP. E-mail: martavs@ @cav.unesp.br. Autor para correspondência.

${ }^{2}$ Zootecnista, mestrado em Zootecnia.

${ }^{3}$ Biólogo do Centro de Aquicultura da UNESP - CAUNESP, doutorado em Patologia. Recebido para publicação em 13.09.00. Aprovado em 30.01.01
} 
relataram que altas doses de vitamina $\mathrm{C}$ $(2.000 \mathrm{mg} / \mathrm{kg})$ e E $(800 \mathrm{mg} / \mathrm{kg})$ diminuíram a mortalidade em peixes infectados com Yersinia ruckeri e Ichthyophthirius multifiliis. O mecanismo pelo qual o peixe elimina ou evita a doença parasitária ainda não está claro.

O presente trabalho teve como objetivo avaliar os efeitos da adição de vitamina $\mathrm{C}$ e $\mathrm{E}$ na dieta sobre o crescimento e sobrevivência de girinos de rã-touro, Rana catesbeiana, em condições experimentais.

\section{MATERIAL E MÉTODOS}

O experimento foi realizado no Laboratório de Nutrição de Organismos Aquáticos do Centro de Aquicultura da UNESP, Jaboticabal, SP no período de 01 de abril a 26 de maio de 1999. Foram testados três níveis de vitamina $\mathrm{C}$ revestida com etilcelulose - Roche ${ }^{\circledR}(50,250$ e $500 \mathrm{mg} / \mathrm{kg})$ e três níveis de vitamina E - Roche ${ }^{\circledR}(50,250$ e $500 \mathrm{mg} / \mathrm{kg}$ ), utilizando-se delineamento inteiramente casualizado, com 7 tratamentos (3 níveis de vitamina C, 3 níveis de vitamina $E$ e ração controle) e 3 repetições, totalizando 21 parcelas.

Os tratamentos foram os seguintes: $\mathrm{T}_{1}$ ração controle $(\mathrm{RC})+50 \mathrm{mg}$ vit $\mathrm{C} / \mathrm{kg} ; \mathrm{T}_{2}-\mathrm{RC}+$ $250 \mathrm{mg}$ vit $\mathrm{C} / \mathrm{kg} ; \mathrm{T}_{3}-\mathrm{RC}+500 \mathrm{mg}$ vit $\mathrm{C} / \mathrm{kg} ; \mathrm{T}_{4}-\mathrm{RC}+$ $50 \mathrm{mg}$ vit $\mathrm{E} / \mathrm{kg} ; \mathrm{T}_{5^{-}} \mathrm{RC}+250 \mathrm{mg}$ vit $\mathrm{E} / \mathrm{kg} ; \mathrm{T}_{6}-\mathrm{RC}+$ $500 \mathrm{mg}$ vit $\mathrm{E} / \mathrm{kg}$ e $\mathrm{T}_{7^{-}}$RC. Foram utilizados 945 girinos de rã-touro (R. catesbeiana), com peso médio inicial de $0,0135 \mathrm{~g}$, os quais foram distribuídos em 21 aquários com volume de 90 litros de água, na densidade de 0,5 girino/ $\ell$ de água, sendo portanto cada parcela constituída de 45 girinos.

Todas as rações experimentais apresentaram a mesma formulação, diferindo apenas no teor de vitamina $\mathrm{C}$ ou $\mathrm{E}$ (Tabela 1). A ração foi oferecida na forma peletizada e lançada ao fundo do aquário, de modo uniforme, duas vezes ao dia, evitando-se sobras, de forma que a quantidade oferecida pudesse ser considerada como consumida (UTNE, 1978). Antes da alimentação os aquários foram sifonados, a fim de retirar os dejetos e restos de ração da alimentação anterior.

O desempenho de produção dos girinos (ganho de peso e sobrevivência) foi avaliado quinzenalmente através da pesagem individual de $20 \%$ dos girinos de cada aquário experimental, com exceção da primeira e da última na qual todos os girinos foram pesados, utilizando-se para isso balança eletrônica digital com sensibilidade de $0,01 \mathrm{~g}$. Os valores de sobrevivência observados para os girinos de rã-touro, por serem expressos em
Tabela 1 - Fórmula e composição percentual da ração controle.

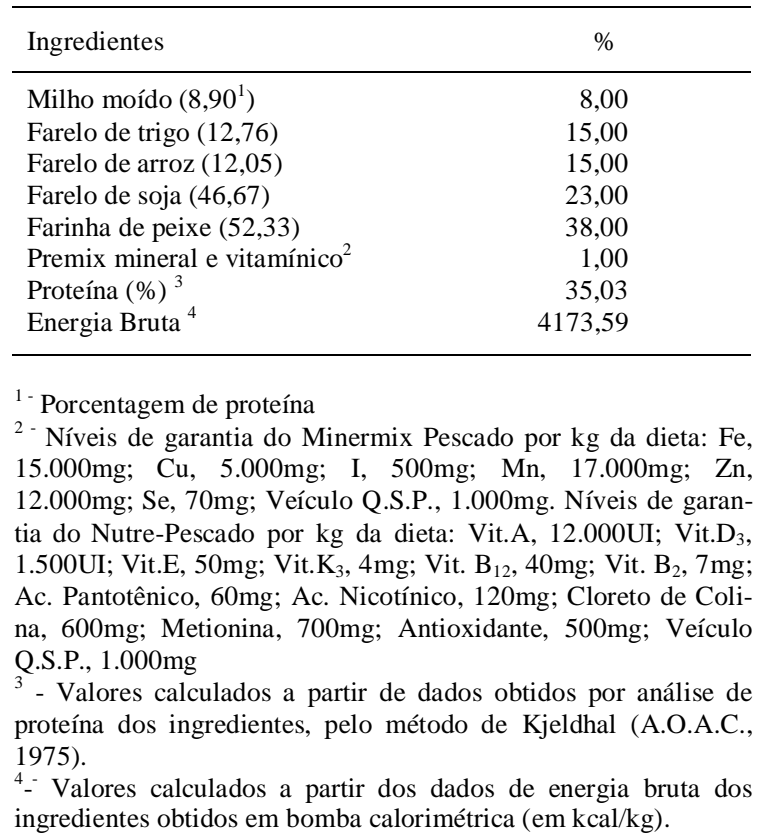

porcentagem, foram transformados para proceder-se à análise de variância em:

$$
\operatorname{arcsen} \sqrt{\frac{p}{100}}
$$

Durante o experimento, os parâmetros aquáticos foram os seguintes: temperatura da água 28,0 a $31,5^{\circ} \mathrm{C} ; \mathrm{pH} 7,10$ a $8,62(7,86 \pm 0,76)$; oxigênio dissolvido 2,96 a $6,64 \mathrm{mg} / \ell \quad(4,80 \pm 1,84)$ e condutividade elétrica 180 a $200 \mu \mathrm{S} / \mathrm{cm}$ $(190,00 \pm 10,00)$.

\section{RESULTADOS E DISCUSSÃO}

Os valores dos parâmetros aquáticos mantiveram-se dentro dos limites aceitáveis para cultivo de organismos aquáticos (SIPAÚBATAVARES, 1994). Com relação ao ganho de peso, não ocorreu diferença significativa entre os tratamentos (Tabela 2), indicando que a suplementação com vitamina $\mathrm{C}$ ou $\mathrm{E}$ não afetou o ganho de peso dos girinos. Resultados semelhantes foram obtidos por LEIBOVITZ et al. (1982) que não observaram diferença no ganho de peso de girinos de rã-touro alimentados com ração suplementada com 0, 2, 4 ou 8\% de vitamina C. Entretanto, MARTINS (1995) observou que a suplementação de vitamina $\mathrm{C}$ melhorou o ganho de peso do pacu $(\boldsymbol{P}$. 
Tabela 2 - Valores de F, coeficiente de variação (CV) e médias dos dados de ganho de peso (g) e sobrevivência (\%) nos diferentes tratamentos durante o período experimental.

\begin{tabular}{ccc}
\hline \multirow{2}{*}{ Estatísticas } & \multicolumn{2}{c}{ Variáveis } \\
\cline { 2 - 3 } & Ganho de Peso (g) & Sobrevivência (\%) \\
\hline F para tratamentos & 2,01 & $2,97 *$ \\
CV (\%) & 10,46 & 15,05 \\
$\mathrm{~T}_{1}-(\mathrm{RC}+50 \mathrm{mg}$ Vit.C/kg) & 5,50 & $44,39 \mathrm{ab}$ \\
$\mathrm{T}_{2}-(\mathrm{RC}+250 \mathrm{mg}$ Vit.C/kg) & 6,60 & $52,38 \mathrm{ab}$ \\
$\mathrm{T}_{3}-(\mathrm{RC}+500 \mathrm{mg}$ Vit.C/kg) & 7,01 & $62,83 \mathrm{a}$ \\
$\mathrm{T}_{4}-(\mathrm{RC}+50 \mathrm{mg}$ Vit.E/kg) & 6,30 & $52,20 \mathrm{ab}$ \\
$\mathrm{T}_{5}-(\mathrm{RC}+250 \mathrm{mg}$ Vit.E/kg) & 5,90 & $58,95 \mathrm{ab}$ \\
$\mathrm{T}_{6}-(\mathrm{RC}+500 \mathrm{mg}$ Vit.E/kg) & 5,89 & $50,15 \mathrm{ab}$ \\
$\mathrm{T}_{7}-(\mathrm{Ração}$ Controle - RC) & 5,75 & $40,48 \mathrm{~b}$ \\
\hline
\end{tabular}

Médias seguidas de mesma letra na coluna não diferem estatisticamente entre si pelo teste de Tukey, a 5\% de probabilidade. NS - não significativo

* - significativo ao nível de 5\% de probabilidade

mesopotamicus), determinando como nível ótimo $139 \mathrm{mg} / \mathrm{kg}$ de ração

A sobrevivência dos girinos que receberam ração suplementada com 500mg de vitamina $\mathrm{C} / \mathrm{kg}$ de ração $(62,83 \%)$ foi significativamente maior $(\mathrm{p}<0,05)$ do que o controle $(40,48 \%)$, indicando que esta suplementação de vitamina $\mathrm{C}$ nas rações de girinos melhorou a sobrevivência dos mesmos (Tabela 2). Entre os demais tratamentos não houve diferença significativa com relação à taxa de sobrevivência. LEIBOVITZ et al. (1982) não observaram diferença na sobrevivência de girinos de rã-touro suplementados com diferentes níveis de vitamina $\mathrm{C}$.

Observa-se que a quantidade de $50 \mathrm{mg}$ de vitamina $\mathrm{E}$ contida no premix vitamínico fornecido aos girinos (Tabela 1), bem como a suplementação da mesma nos níveis testados não influenciaram o ganho de peso e taxa de sobrevivência dos mesmos.

$\mathrm{O}$ presente trabalho é o primeiro relato sobre suplementação com vitamina C e E na alimentação de girinos de rã-touro. Por outro lado, estudos futuros devem ser realizados, uma vez que tem sido comprovado que a utilização dessas vitaminas vem contribuindo para melhor crescimento e aumento na resistência a doenças em peixes.

\section{CONCLUSÃO}

Nas condições em que o experimento foi realizado e com base nos dados apresentados, podese concluir que a adição de vitamina $\mathrm{C}$ aumentou a sobrevivência dos girinos e a adição de vitamina $\mathrm{E}$, nas doses testadas, não alterou o desempenho dos mesmos.

\section{REFERÊNCIAS BIBLIOGRÁFICAS}

ASSOCIATION OF OFFICIAL ANALITICAL CHEMISTS AOAC. Officcial methods of analysis. 12.ed. Washington, 1975. 1094p.

AGRAWAL, N.K., MAHAJAN, C.L. Nutritional deficiency disease in an Indian major carp Cirrhina mrigala $\mathrm{H}$., due to avitaminosis C during early growth. Journal of Fish Diseases, v.3, p.231-248, 1980.

HALVER, J.E., SMITH, R.R., TOLBERT, B.M., et al. Utilization of ascorbic acid in fish. Annual New York Academy of Science, v.258, p.81-102, 1975.

HALVER, J.E. Vitamim requeriment study techniques. J. Applied Ichthyology, v.11, p.215-228, 1995.

LEIBOVITZ, H.E., CULLEY Jr., D.D.,GEAGHAN, J.P. Effects of vitamin $\mathrm{C}$ and sodium benzoate on survival, growth and skeletal deformities of intensively cultured bullfrog larvae Rana catesbeiana reared at two $\mathrm{pH}$ levels. J ournal of the World Mariculture Society, v.13,p.322-328, 1982.

MARTINS, M.L. Effect of ascorbic acid deficiency on the growth, gill filament lesions and behaviour of pacu fry (Piaractus mesopotamicus Holmberg, 1887). Brazilian Journal of Medical and Biological Research, v.28, p.563$568,1995$.

MARTINS, M.L. Evaluation of the addition of ascorbic acid to the ration of cultivated Piaractus mesopotamicus (Characidae) on the infrapopulation of Anacanthorus penilabiatus (Monogenea). Brazilian Journal of Medical and Biological Research, v.31, p.655-658, 1998.

SIPAÚBA-TAVARES, L.H. Limnologia aplicada à Aquicultura. Funep/UNESP, 1994. 70p. (Boletim Técnico $\mathrm{n}^{\mathrm{o}} 1$ ).

UTNE, F. Standard methods and therminology in finfish nutrition. In: SIMPOSIUM OF FINFISH NUTRITION AND FISH FEED TECHNOLOGY, 1978, Hamburg. Proceedings... Hamburg : EIFAC/FAO,1978. R-1. 14p.

WAHLI, T., VERLHAC, V., GABAUDAN, J., et al. Influence of combined vitamin $\mathrm{C}$ and $\mathrm{E}$ on non-specific immunity and disease resistance of rainbow trout, Oncorhynchus mykiss (Walbaum). Journal of Fish Diseases, v.21, p.127-137, 1998. 